\title{
INFLUENCES OF PRE-SEGMENTAL ARTERY VARIATION ON MICRO-KIDNEY: A CASE REPORT
}

\author{
Eren Ogut ${ }^{1}$, Mert Sonkaya $^{2}$, Cagatay Barut ${ }^{1}$
}

${ }^{1}$ Department of Anatomy, Bahcesehir University, Faculty of Medicine, 34734 İstanbul, TURKEY

2 Medical Faculty Student, School of Medicine, Bahcesehir University, 34734 İstanbul, TURKEY

\author{
Address for Correspondence: Prof. Dr. Cagatay Barut, E-mail: cagbarut@yahoo.com \\ Received: 04.01.2021; Accepted: 28.01.2021; Available Online Date: 04.03.2021 \\ (C)opyright 2020 by Dokuz Eylül University, Institute of Health Sciences - Available online at https://dergipark.org.tr/tr/pub/jbachs
}

Cite this article as: Ogut E, Sonkaya M, Cagatay B. Influences of pre-segmental artery variation on micro-kidney: A case report. J Basic Clin Health Sci 2021; 1: 78- 81.

\begin{abstract}
We present a case with accessory pre-segmental arteries and micro-kidneys. There are no articles on the effects of the accessory pre-segmental renal artery on the size of the kidney. Therefore this study aimed to investigate whether a relationship exists between the accessory arteries and kidney size. Left presegmental artery variations and bilateral microkidneys were detected in a 62-year-old female cadaver during routine retroperitoneal dissection. The right micro kidney was $7.74 \mathrm{~cm}$ in length, $3.82 \mathrm{~cm}$ in width and $3.87 \mathrm{~cm}$ in thickness, and the left micro kidney was $6.82 \mathrm{~cm}$ in length, $3.42 \mathrm{~cm}$ in width and $3.02 \mathrm{~cm}$ in thickness. The superior left pre-segmental artery $\mathrm{L} 1$ at the origin site of the left renal artery and just below the superior mesenteric artery was $8.98 \mathrm{~cm}$ in length, $0.54 \mathrm{~cm}$ in width and $0.42 \mathrm{~cm}$ in thickness, and the inferior left pre-segmental artery L1-L2 at the origin site of the left renal artery was $8.92 \mathrm{~cm}$ in length, $0.54 \mathrm{~cm}$ in width and $0.41 \mathrm{~cm}$ in thickness. The presence of the accessory pre-segmental artery might provide clues regarding the presence of an abnormality of the kidney. Therefore, surgeons should know pre-segmental artery variations and associated kidney size abnormalities.
\end{abstract}

Keywords: Renal artery, pre-segmental artery, variation, micro-kidney, kidney

\section{INTRODUCTION}

The renal arteries divide into pre-segmental arteries, which further divide into segmental arteries providing the renal arterial segments $(1,2)$. The segmental branches of the renal artery have early division, hilar (accessory), and polar arteries. Hilar arteries intrude into the kidneys from the hilus with the central renal artery, whereas polar arteries join the kidneys directly from the capsule out of the hilus (1). Awareness of accessory renal artery variations and associated conditions is essential for planning surgical procedures, since variations in the number, origin, and route of the renal arteries are frequent, including the accessory renal artery (3). The arterial variations of the kidney derive from the embryological progress of renal arteries during fetal development and lead to 
abnormal positions and size of kidneys in adult life. Therefore, the presence of pre-segmental artery variations might lead to abnormal kidney development and changed the positions and size of the kidneys. Accessory renal arteries usually originate from the abdominal aort, renal artery, celiac trunk, superior mesenteric and inferior mesenteric, common iliac, middle sacral, or external iliac arteries, and they are called tenacious embryonic lateral splanchnic arteries (3). Several authors have reported that the accessory renal arteries cause kidney abnormalities and various conditions such as nephrosclerosis, atherosclerosis, glomerulosclerosis, interstitial fibrosis, tubular atrophy, and kidney size abnormalities $(2,4,5)$. Therefore, the presence of the accessory pre-segmental artery might provide clues regarding the presence of an abnormality of the kidney.

\section{CASE REPORT}

Left pre-segmental artery variations and bilateral micro-kidneys were identified in a 62-year-old female cadaver with unknown disease during routine retroperitoneal dissection. The incidence, length, width, thickness, and location of the pre-segmental arteries and kidneys were measured with a $150 \mathrm{~mm}$ digital vernier dial microcaliper. Photographs were taken by Canon EOS 70D using a $100 \mathrm{~mm}$ macro lens. The artery and kidney variations were recorded, and photographs were taken as defined by Barut and Ertilav (6). The right micro kidney was $7.74 \mathrm{~cm}$ (length), $3.82 \mathrm{~cm}$ (width) and $3.87 \mathrm{~cm}$ (thickness), the left micro kidney was $6.82 \mathrm{~cm}$ (length), $3.42 \mathrm{~cm}$ (width) and $3.02 \mathrm{~cm}$ (thickness) with unilateral left pre-segmental artery variations. The superior left presegmental artery ( $L 1$, at the origin site of the left renal artery, and just below the superior mesenteric artery) was $8.98 \mathrm{~cm}$ (length), $0.54 \mathrm{~cm}$ (width), $0.42 \mathrm{~cm}$ (thickness), and the inferior left pre-segmental artery L1-L2 at the origin site of the left renal artery was 8.92 $\mathrm{cm}$ (length), $0.54 \mathrm{~cm}$ (width), and $0.41 \mathrm{~cm}$ (thickness) (Figure 1). The accessory pre-segmental artery entered through the surface of the kidney below its hilum. Variations of the pre-segmental artery were seen in the origin of the left renal artery and extra-hilar site of the left kidney. Moreover, a 78-year-old female cadaver with unknown disease also had an accessory right renal artery (with normal size kidneys)

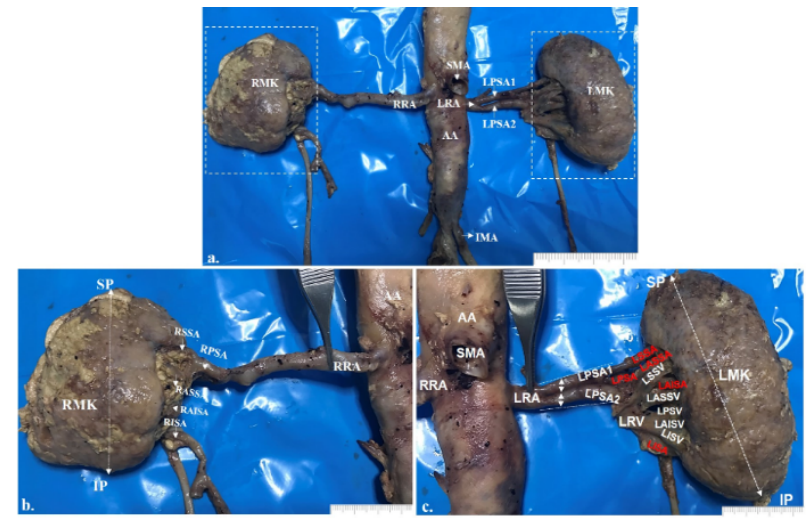

originating from the abdominal aort at the level of $L 3$ and entering through the kidney's inferior pole (Figure 2).

Figure 1. a. Bilateral micro (hypoplastic) kidney in female cadaver with left pre-segmental renal artery variations (anterior view). AA: Abdominal Aort; SMA: Superior Mesenteric Artery (white arrow); RRA: Right Renal Artery; LRA: Left Renal Artery (white arrow); LPSA1: Left pre-segmental artery 1 (white arrow); LPSA2: Left presegmental artery 2 (white arrow) b. Right Micro Kidney (RMK) \& normal right pre-segmental renal artery. AA: Abdominal Aort; RRA: Right Renal Artery; SP: Superior Pole; IP: Inferior Pole; RPSA: Right pre-segmental artery (white arrow); RSSA: Right Superior Segmental Artery (arrowhead); RASSA: Right AnteroSuperior Segmental Artery (white arrow); RAISA: Right Anteroinferior Segmental Artery (white arrow); RISA: Right Inferior Segmental Artery (white arrow) c. Left micro-kidney with accessory presegmental artery Micro (hypoplastic) kidney with an accessory presegmental artery on the left side (anterior view). AA: Abdominal Aort; SMA: Superior Mesenteric Artery; RRA: Right Renal Artery; LRA: Left Renal Artery; SP: Superior Pole; IP: Inferior Pole; LPSA1: Left pre-segmental artery 1; LPSA2: Left pre-segmental artery 2; LRV: Left Renal Vein; LPSA: Left Posterior Segmental Artery; LSSA: Left Superior Segmental Artery; LASSA: Left AnteroSuperior Segmental Artery; LAISA: Left Anteroinferior Segmental Artery; LISA: Left Inferior Segmental Artery; LSSV: Left Superior Segmental Vein; LASSV: Left AnteroSuperior Segmental Vein; LAISA: Left Anteroinferior Segmental Vein; LPSV: Left Posterior Segmental Vein; LISV: Left Inferior Segmental Vein.

\section{DISCUSSION}

Several origins of accessory renal arteries and variations are due to the development process of mesonephric arteries, and it can result in more than one renal artery (1). Sasikala P et al. (2) stated that the accessory renal artery was linked to congenital kidney anomalies in two cadavers. One of the cadavers had polycystic kidney disease with an accessory renal artery on the right side, and the other cadaver had a malrotated kidney with an accessory renal artery on the left side. Sasikala P et al (2). stated that the accessory renal artery could be due to the abnormal development of kidneys and variations in 
the kidney's position. An accessory renal artery with unilateral congenital anomalies is more frequent than bilateral abnormalities. Dhar et al. found an unusual renal artery with a unilateral anomaly in $15 \%$ of cases and bilateral aberration in 5\% (4). Guang-Qian Xiao et al. (7) described a unilateral hypoplastic kidney in a 33-year-old male patient. Likewise, Sasilkala et al. described one hypoplastic kidney out of eight kidneys with an accessory renal artery on the right side.

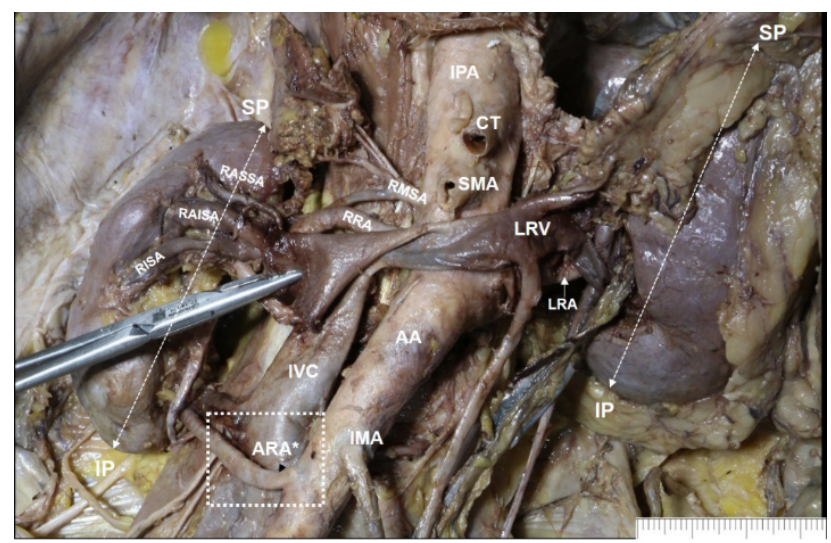

Figure 2. Normal kidney with an accessory renal artery on the right side. Right accessory renal artery (L3) CT: Coeliac Trunk; IPA: Inferior Phrenic Artery (white arrow); IVC: Inferior Vena Cava; SMA: Superior Mesenteric Artery; IMA: Inferior Mesenteric Artery; ARA*: Accessory Renal Artery (Right) (white arrow); LRA; Left Renal Artery (white arrow); SP: Superior Pole; IP: Inferior Pole; LRV: Left Renal Vein; RASSA: Right AnteroSuperior Segmental Artery (white arrow); RAISA: Right Anteroinferior Segmental Artery; RISA: Right Inferior Segmental Artery; RRA: Right Renal Artery; RMSA: Right Middle Supraenal Artery.

In the current study, we detected a bilateral kidney anomaly (micro-kidney) with an accessory presegmental artery (unilateral accessory presegmental artery anomaly) on the left side of female cadever. Kidneys develop symmetrically, through fetal development, but the left kidney was more extensive than the right kidney. This might have been due to the presence of multiple artery variations (8). Sosnik $\mathrm{K}$ et al. (8) indicated that the mean size of the kidney is significantly dependent on the number of renal arteries. Kidneys with multiple arteries also show a specific relationship, although this is irrelevant to the length of renal arteries branching from the aorta (8). Glodny et al. (5) claimed that the kidney size depends on the number of arteries; therefore, the renal artery variations associated to congenital irregularities are essential to managing renal trauma, renal transplantation, renovascular hypertension, renal 80 artery embolization, angioplasty and vascular reconstruction for congenital or acquired lesions. Sarfraz R et al. (9) stated the variations of presegmental arteries with the number and sites of origin to be as follows: $75.7 \%$ were extra-hilar, $21.6 \%$ were hilar, and $2.7 \%$ were intrarenal. In the present study, a 62-year-old female cadaver had bilateral microkidneys with the left accessory pre-segmental extrahilar arteries which originated from the left renal artery's position at the level of $L 1$, just below the superior mesenteric artery. Macchi $\mathrm{V}$ et al (10) reported that the presence of a collateral arterial supply between the segments of the kidney should be taken into account, particularly when a selective clamping is planned. Furthermore, the presence of accessory vessels between the segments can decrease the potential ischaemic damage to the renal parenchyma and haemorrhagic complications during surgery (10). Therefore, pre-segmental artery variations are taken into consideration for diagnostic investigations and to avoid complications during surgical approaches to the retroperitoneal region.

\section{CONCLUSION}

In conclusion, the presence of pre-segmental artery variations might associated with abnormal kidney size or development. Measurement of the accessory presegmental artery might provide clues regarding the presence of an abnormality of the kidney.

Conflict of Interest: No conflict of interest was declared by the authors.

Authors' contributions: $\mathrm{BC}$ and $\mathrm{OE}$ contributed to the data analysis and manuscript writing. $\mathrm{BC}$ and $\mathrm{OE}$ conceived the project and contributed to final manuscript approval. All authors read and approved the final manuscript.

\section{REFERENCES}

1. Ozkan U, Oğuzkurt L, Tercan F, Kizilkiliç O, Koç $Z$, Koca N. Renal artery origins and variations: angiographic evaluation of 855 consecutive patients. Diagn Interv Radiol. 2006;12(4):183-6.

2. Sasikala $P$, Singh $S$. Accessory renal artery associated with congenital kidney anomalies. Anatomy. 2017;11(2):67-71.

3. Bannister LH, Berus MM, Collins P, Dyson M, Dusek JE, Ferguson MWJ. Gray's anatomy. 40th ed. Edinburgh: Churchill Livingstone; 2008. p. 
1225-33. 40th ed. ed. Edinburgh: Churchill Livingstone2008.

4. Dhar P, Lal K. Main and accessory renal arteries-a morphological study. Ital J Anat Embryol. 2005;110(2):101-10.

5. Glodny B, Unterholzner V, Taferner B, Hofmann KJ, Rehder $P$, Strasak A, et al. Normal kidney size and its influencing factors - a 64-slice MDCT study of 1.040 asymptomatic patients. BMC Urol. 2009;9:19.

6. Barut C, Ertilav H. Guidelines for standard photography in gross and clinical anatomy. Anat Sci Educ. 2011;4(6):348-56.

7. Xiao GQ, Jerome JG, Wu G. Unilateral hypoplastic kidney and ureter associated with diverse mesonephric remnant hyperplasia. Am J Clin Exp Urol. 2015;3(2):107-11.

8. Sośnik H. Does Kidney Size Depend on the Number of Renal Arteries? EC Clinical and Experimental Anatomy 2019;2(3):82-9.

9. Sarfraz R, Tahir M, Sami W. Presegmental Arterial Pattern of Human Kidneys in Local Population. Ann Pak Inst Med Sci 2008;4(4):2125.

10. Macchi V, Crestani A, Porzionato A, Sfriso MM, Morra A, Rossanese M, et al. Anatomical study of renal arterial vasculature and its potential impact on partial nephrectomy. BJU Int. 2017;120(1):8391. 